\title{
Natural Frequency Analysis of Two Nonlinear Panels Coupled with a Cavity Using the Approximate Elliptic Integral Solution and the Method of Harmonic Residual Minimization
}

\author{
Y.Y. Lee \\ Department of Civil and Architectural Engineering, City University of Hong Kong, Kowloon Tong, Kowloon, Hong Kong \\ Correspondence should be addressed to Y. Y. Lee; bcraylee@cityu.edu.hk
}

Received 8 April 2014; Revised 21 October 2014; Accepted 3 November 2014

Academic Editor: Zengji Du

Copyright (C) 2015 Y. Y. Lee. This is an open access article distributed under the Creative Commons Attribution License, which permits unrestricted use, distribution, and reproduction in any medium, provided the original work is properly cited.

\begin{abstract}
The nonlinear structural acoustic problem considered in this study is the nonlinear natural frequency analysis of flexible double panels using the elliptic integral solution method. There are very limited studies for this nonlinear structural-acoustic problem, although many nonlinear plate or linear double panel problems have been tackled and solved. A multistructural/acoustic modal formulation is derived from two coupled partial differential equations which represent the large amplitude structural vibrations of the flexible panels and acoustic pressure induced within the air gap. One is the von Karman's plate equation and the other is the homogeneous wave equation. The results obtained from the proposed method approach are verified with those from a numerical method. The effects of vibration amplitude, gap width, aspect ratio, the numbers of acoustic modes and harmonic terms, and so forth on the resonant frequencies of the in-phase and out of phase modes are examined.
\end{abstract}

\section{Introduction}

In practice, thin double panels are very common for sound reduction. As it is easy to get thin panels excited nonlinearly, the nonlinear effect on the resonant frequencies of double panel is studied in this paper. However, there are very limited studies for this nonlinear structural-acoustic problem, although many nonlinear plate or linear double panel problems have been tackled and solved. Thus, the following paragraph would be the reviews about linear double panel and nonlinear panel vibration.

Over the past decades, many researchers such as London [1], Cummings and Mulholland [2], and Price and Crocker [3] studied double panels and adopted the multiple-reflection theory and statistical energy analysis to develop various formulas for prediction. In their works, the panel sizes were assumed very large so that the first few structural resonant frequencies were far below $50 \mathrm{~Hz}$ and not concerned. Thus, the concept of the mass-cavity-mass, which ignored the panel stiffness, was valid for common audible frequency range. The statistical energy method was suitable for cases of high modal density or high frequency. If a smaller panel is considered, the first few fundamental resonant frequencies would move to a higher frequency range (say $80-150 \mathrm{~Hz}$ ). Under this condition, the bending stiffness of the panel cannot be ignored, and the statistical energy method would not be suitable. In the works of Xin and Lu [4-7] and Xin et al. [810], the detailed theoretical and experimental investigations about the sound absorptions, radiations, and vibrations of various sandwich panels and double panels were included. In some of their works, the solution method adopted was suitable for double panel systems of finite or infinite extent and applicable for both low- and high-frequency ranges with these merits; the proposed method compares favorably with a number of other approaches. Besides, it was pointed out that the structural-acoustic coupling affected significantly the sound and vibration behaviors at low frequencies and could not be ignored. Pan and Bao [11] and Kaiser et al. [12] adopted the modal analysis to develop their theoretical models for studying a linear double panel problem at low frequencies. Langer and Antes [13] studied double walls with different pane configurations using the finite and boundary element methods. In $[14,15]$, the works considered the resonant frequencies of the first two modes of the structural-acoustic 


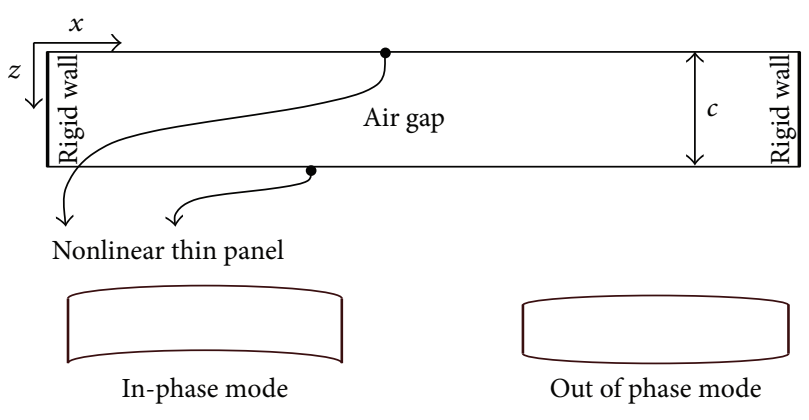

Figure 1: A double panel and its mode shapes.

problem. It was proven experimentally and theoretically in these works that the first two modes could seriously deteriorate the noise reduction performance. That was an important finding. Hence, the in-phase and out of phase modes of double panels are the focus of this paper. The structural-acoustic models in the aforementioned works were capable of predicting multimode responses, but not the large amplitude vibration effect, and the modal coupling effects on the resonant frequencies were not investigated. There are very limited studies for nonlinear double panel problems. Some studies (e.g., [16-24]) are related to the nonlinear structural vibrations, but their focuses are different from that in this paper. In fact, there are numerous theories used for solving various nonlinear vibration problems (e.g., [25-35]). In some approaches for solving the problems of large amplitude structure or nonlinear oscillation (e.g., [36-39]), they require a significant effort in the tedious eigenvalue solution steps. The present study uses the proposed elliptical integral method to develop a concise elliptical integral solution for the large amplitude free vibration analysis.

\section{Theoretical Formulation}

According to the formulation of Chu and Herrmann [25], the governing equation for the large amplitude vibration of a rectangular plate is given by

$$
\rho \frac{d^{2} A}{d t^{2}}+\rho \omega_{p}^{2} A+\beta A^{3}=0 .
$$

Note that in the studies of [23-25], the panel vibration amplitude ranges are zero to $1.4 h$, where $h=$ panel thickness. Within this vibration amplitude range, no panel buckling occurs.

Consider the acoustic pressures within the gap acting on the two panels (see Figure 1). Equation (1a) is modified and given by

$$
\begin{aligned}
& \rho \frac{d^{2} A}{d t^{2}}+\rho \omega_{p}^{2} A+\beta A^{3}+F_{c}=0, \\
& \rho \frac{d^{2} B}{d t^{2}}+\rho \omega_{p}^{2} B+\beta B^{3}-F_{o}=0,
\end{aligned}
$$

where $A(t)$ and $B(t)$ are the modal displacements of the two panels at $z=0$ and $c$. The mode shapes of the two panels are $\phi(x, y)=\sin (\pi x / a) \sin (\pi y / b) ; t$ is time; $\rho$ is the panel surface density; $F_{c}$ and $F_{o}$ are the modal pressure force induced by the panel vibration; $\omega_{p}=\sqrt{E h^{2} / 12 \rho\left(1-v^{2}\right)}\left((\pi / a)^{2}+(\pi / b)^{2}\right)$ are the fundamental linear natural frequencies of the panels; $\beta=$ $\left(E h / 12\left(1-\nu^{2}\right)\right)\left(\gamma / a^{4}\right)$ is the nonlinear stiffness coefficient that is due to the large amplitude vibration; $E$ is Young's modulus of the panels; $\gamma=3 \pi^{4}\left[\left((3 / 4)-\left(\nu^{2} / 4\right)\right)\left(1+r^{4}\right)+\nu r^{2}\right] ; r=a / b$ is the aspect ratio; $v$ is Poisson's ratio; and $a$ and $b$ are the panel length and width.

$A(t)$ and $B(t)$ are periodic and considered to be a summation of harmonic terms and are given by

$$
\begin{gathered}
A(t)=\sum_{h=1,3,5, \ldots}^{H} A^{h}(t), \\
B(t)=\sum_{h=1,3,5, \ldots}^{H} B^{h}(t),
\end{gathered}
$$

where $A^{h}$ and $B^{h}$ are the $h$ th-order harmonic responses and $H=$ the number of harmonic terms used.

Similarly, the total modal acoustic pressure forces acting on the panel surfaces are given by

$$
\begin{aligned}
& F_{c}(t)=\sum_{h=1,3,5, \ldots}^{H} P_{c}^{h}(t), \\
& F_{o}(t)=\sum_{h=1,3,5, \ldots}^{H} P_{o}^{h}(t),
\end{aligned}
$$

where $P_{c}^{h}(t)$ and $P_{o}^{h}(t)$ are the acoustic pressure forces induced by the $h$ th-order harmonic components of the two nonlinear panel vibrations, respectively.

In Figure 1, the acoustic pressure within the gap induced by the flexible enclosure panel and panel absorber is given by the following homogeneous wave equation $[14,15]$ :

$$
\nabla^{2} P^{h}-\frac{1}{C_{a}^{2}} \frac{\partial^{2} P^{h}}{\partial t^{2}}=0
$$

where $P^{h}$ is the acoustic pressure within the gap induced by the $h$ th harmonic component of the nonlinear panel vibration and $C_{a}$ is the speed of sound.

The boundary conditions of the air gap are

$$
\begin{gathered}
\frac{\partial P^{h}}{\partial x}=0 \quad \text { at } x=0, a \\
\frac{\partial P^{h}}{\partial y}=0 \quad \text { at } y=0, b \\
\frac{\partial P^{h}}{\partial z}=-\rho_{a} \frac{\partial^{2} w_{o}^{h}(x, y, t)}{\partial t^{2}} \text { at } z=0 \\
\frac{\partial P^{h}}{\partial z}=-\rho_{a} \frac{\partial^{2} w_{c}^{h}(x, y, t)}{\partial t^{2}} \text { at } z=c,
\end{gathered}
$$

where $a$ and $b$ are the panel width and length, respectively; $c$ is the gap width; $\rho_{a}=$ air density, $w_{o}^{h}(x, y, t)$, and $w_{c}^{h}(x, y, t)$ 
are the $h$ th harmonic components of the nonlinear panel displacements at $z=0$ and $c$, respectively, which can be rewritten as

$$
\begin{array}{ll}
w_{c}^{h}(x, y, t)=A^{h}(t) \phi(x, y) & \text { at } z=c \\
w_{o}^{h}(x, y, t)=B^{h}(t) \phi(x, y) & \text { at } z=0,
\end{array}
$$

where

$$
\begin{aligned}
& w_{c}(x, y, t)=\sum_{h=1,3,5, \ldots}^{H} w_{c}^{h}(x, y, t)=\sum_{h=1,3,5, \ldots}^{H} A^{h}(t) \phi(x, y), \\
& w_{o}(x, y, t)=\sum_{h=1,3,5, \ldots}^{H} w_{o}^{h}(x, y, t)=\sum_{h=1,3,5, \ldots}^{H} B^{h}(t) \phi(x, y),
\end{aligned}
$$

$w_{o}(x, y, t)$ and $w_{c}(x, y, t)$ are the $h$ th harmonic components of the nonlinear panel displacements at $z=0$ and $c$, respectively. $\phi(x, y)=\sin ((\pi / a) x) \sin ((\pi / b) y)$ is the panel mode shape.

According to $[14,15]$, the general multiacoustic mode solution of (1a)-(1c) is

$$
\begin{aligned}
P^{h}=\sum_{u}^{U} \sum_{v}^{V}\left(L_{u v}^{h} \sinh \left(\mu_{u v}^{h} z\right)\right. \\
\left.+N_{u v}^{h} \cosh \left(\mu_{u v}^{h} z\right)\right) \varphi_{u v}(x, y) T(t),
\end{aligned}
$$

where $\mu_{u v}^{h}=\sqrt{(u \pi / a)^{2}+(v \pi / b)^{2}-\left(h \omega / C_{a}\right)^{2}} ; \varphi_{u v}(x, y)=$ $\cos ((u \pi / a) x) \cos ((v \pi / b) y)$ is the acoustic mode; $u$ and $v$ are the acoustic mode numbers; $\omega$ is the excitation frequency. $L_{u v}^{h}$ and $N_{u v}^{h}$ are coefficients that depend on the boundary conditions at $z=0$ and $z=c$; $U$ and $V$ are the numbers of acoustic mode numbers used. $C_{a}$ is sound speed. $T(t)$ is the time function.

By applying the boundary conditions in (2c) and (2d) to (5a) and (5b), the unknown coefficients, $L_{u v}^{h}$ and $N_{u v}^{h}$, can be found, and thus the $h$ th harmonic component of the modal internal pressure forces within the gap at $z=c$ and 0 (i.e., $P_{c}^{h}$ and $P_{o}^{h}$ ) is given by

$$
\begin{aligned}
& P_{c}^{h}=K_{A}^{h} A^{h}-K_{B}^{h} B^{h}, \\
& P_{o}^{h}=K_{B}^{h} A^{h}-K_{A}^{h} B^{h},
\end{aligned}
$$

where

$$
\begin{gathered}
K_{B}^{h}=\sum_{u}^{U} \sum_{v}^{V} \frac{\rho_{a}(h \omega)^{2}}{\mu_{u v}^{h}} \frac{\left(\alpha_{u v}^{\phi}\right)^{2}}{\alpha_{u v}^{u v} \alpha_{\phi}^{\phi}} \frac{1}{\sinh \left(\mu_{u v}^{h} c\right)} ; \\
K_{A}^{h}=\sum_{u}^{U} \sum_{v}^{V} \frac{\rho_{a}(h \omega)^{2}}{\mu_{u v}^{h}} \frac{\left(\alpha_{u v}^{\phi}\right)^{2}}{\alpha_{u v}^{u v} \alpha_{\phi}^{\phi}} \operatorname{coth}\left(\mu_{u v}^{h} c\right) ;
\end{gathered}
$$

$\alpha_{u v}^{\phi}=\int_{0}^{b} \int_{0}^{a} \varphi_{u v} \phi d x d y ; \alpha_{u v}^{u v}=\int_{0}^{b} \int_{0}^{a} \varphi_{u v} \varphi_{u v} d x d y ; \alpha_{\phi}^{\phi}=$ $\int_{0}^{b} \int_{0}^{a} \phi^{2} d x d y$; $U$ and $V$ are the maximum acoustic mode numbers.

Then, the total modal pressure forces within the gap at $z=c$ and 0 and the external pressure force acting on the panel absorber $\left(F_{c}, F_{o}\right.$, and $\left.F_{e}\right)$ are given by

$$
\begin{aligned}
& F_{c}=\sum_{h=1,3,5, \ldots}^{H} P_{c}^{h}, \\
& F_{o}=\sum_{h=1,3,5, \ldots}^{H} P_{o}^{h},
\end{aligned}
$$

where $H$ is the number of harmonic terms used.

Putting (10a) and (10b) into (1a)-(1b) yields

$$
\begin{aligned}
& \rho \frac{d^{2} A}{d t^{2}}+\rho \omega_{o}^{2} A+\beta A^{3}+\sum_{h=1,3,5, \ldots}^{H} K_{A}^{h} A^{h}-K_{B}^{h} B^{h}=0 \\
& \rho \frac{d^{2} B}{d t^{2}}+\rho \omega_{o}^{2} B+\beta B^{3}+\sum_{h=1,3,5, \ldots}^{H} K_{A}^{h} B^{h}-K_{B}^{h} A^{h}=0 .
\end{aligned}
$$

Note that $A$ and $B$ are the displacement responses of the two panels and given by

$$
\begin{gathered}
A(t)=\sum_{h=1,3,5, \ldots}^{H} A^{h}(t), \\
B(t)=\sum_{h=1,3,5, \ldots}^{H} B^{h}(t) .
\end{gathered}
$$

Figure 1 shows the two modes of the double panel. Then set $A(t)=B(t)$ for the in-phase mode and $A(t)=-B(t)$ for the out of phase mode in (8a) and (8b). Hence, we get the following.

For the in-phase mode

$$
\rho \frac{d^{2} A}{d t^{2}}+\rho \omega_{o}^{2} A+\beta A^{3}+\sum_{h=1,3,5, \ldots}^{H}\left(K_{A}^{h}-K_{B}^{h}\right) A^{h}=0 .
$$

For the out of phase mode

$$
\rho \frac{d^{2} A}{d t^{2}}+\rho \omega_{o}^{2} A+\beta A^{3}+\sum_{h=1,3,5, \ldots}^{H}\left(K_{A}^{h}+K_{B}^{h}\right) A^{h}=0 .
$$

Consider the dummy term $K_{1} A$ and $K_{2} A$ in (13a) and (13b):

$$
\begin{aligned}
\rho \frac{d^{2} A}{d t^{2}} & +\left(\rho \omega_{o}^{2}+K_{1}\right) A+\beta A^{3} \\
& +\sum_{h=1,3,5, \ldots}^{H}\left(K_{A}^{h}-K_{B}^{h}\right) A^{h}-K_{1} A=0, \\
\rho \frac{d^{2} A}{d t^{2}} & +\left(\rho \omega_{o}^{2}+K_{2}\right) A+\beta A^{3} \\
& +\sum_{h=1,3,5, \ldots}^{H}\left(K_{A}^{h}+K_{B}^{h}\right) A^{h}-K_{2} A=0,
\end{aligned}
$$

where $K_{1}$ and $K_{2}$ are unknowns to be determined. 
Then, consider the elliptical integral solutions in [23] for the following equations:

$$
\begin{gathered}
\rho \frac{d^{2} \bar{A}_{1}}{d t^{2}}+\left(\rho \omega_{o}^{2}+K_{1}\right) \bar{A}_{1}+\beta \bar{A}_{1}^{3}=0, \\
\rho \frac{d^{2} \bar{A}_{2}}{d t^{2}}+\left(\rho \omega_{o}^{2}+K_{2}\right) \bar{A}_{2}+\beta \bar{A}_{2}^{3}=0,
\end{gathered}
$$

where $\bar{A}_{1}=A_{1} c n\left(u\left(\kappa_{1}\right)\right)$ and $\bar{A}_{2}=A_{2} c n\left(u\left(\kappa_{2}\right)\right) ; u\left(\kappa_{1}\right)$ and $u\left(\kappa_{2}\right)$ are the elliptic integrals; $\kappa_{1}=\beta A_{1}^{2} / 2\left(\rho \omega_{0}^{2}+K_{1}+\right.$ $\left.\beta A_{1}^{2}\right)$ and $\kappa_{2}=\beta A_{2}^{2} / 2\left(\rho \omega_{0}^{2}+K_{2}+\beta A_{2}^{2}\right)$ are the modulus of $u$; $c n$ is the elliptic cosine; and $A_{1}$ and $A_{2}$ are the modal displacements at $t=0$ or the vibration amplitudes. by

The periods of the nonlinear modal vibrations are given

$$
\begin{aligned}
& T_{1}=\frac{4}{\sqrt{\omega_{0}^{2}+\left(\left(K_{1}+\beta A_{1}^{2}\right) / \rho\right)}} \int_{0}^{\pi / 2} \frac{1}{\sqrt{1-\kappa_{1}^{2} \sin (\psi)}} d \psi, \\
& T_{2}=\frac{4}{\sqrt{\omega_{0}^{2}+\left(\left(K_{2}+\beta A_{2}^{2}\right) / \rho\right)}} \int_{0}^{\pi / 2} \frac{1}{\sqrt{1-\kappa_{2}^{2} \sin (\psi)}} d \psi .
\end{aligned}
$$

Replacing $A_{1}$ with $\bar{A}_{1}$ and $A_{2}$ with $\bar{A}_{2}$ in (14a) and (14b) gives

$$
\begin{aligned}
\rho \frac{d^{2} \bar{A}_{1}}{d t^{2}} & +\left(\rho \omega_{o}^{2}+K_{1}\right) \bar{A}_{1}+\beta \bar{A}_{1}^{3} \\
& +\sum_{h=1,3,5, \ldots}^{H}\left(K_{A}^{h}-K_{B}^{h}\right) \bar{A}_{1}^{h}-K_{1} \bar{A}_{1}=R_{1}, \\
\rho \frac{d^{2} \bar{A}_{2}}{d t^{2}} & +\left(\rho \omega_{o}^{2}+K_{2}\right) \bar{A}_{2}+\beta \bar{A}_{2}^{3} \\
& +\sum_{h=1,3,5, \ldots}^{H}\left(K_{A}^{h}+K_{B}^{h}\right) \bar{A}_{2}^{h}-K_{2} \bar{A}_{2}=R_{2},
\end{aligned}
$$

where $R_{1}$ and $R_{2}$ are the harmonic residuals, as $\bar{A}_{1}$ and $\bar{A}_{2}$ are not the exact solution to (14a) and (14b). $\bar{A}_{1}^{h}$ and $\bar{A}_{2}^{h}$ are the harmonic components of $\bar{A}_{1}$ and $\bar{A}_{2}$.

Using (15a) and (15b), the summation of the first three terms in (17a) and (17b) are set to zero, as follows:

$$
\begin{aligned}
& \sum_{h=1,3,5, \ldots}^{H}\left(K_{A}^{h}-K_{B}^{h}\right) \bar{A}_{1}^{h}-K_{1} \bar{A}_{1}=R_{1}, \\
& \sum_{h=1,3,5, \ldots}^{H}\left(K_{A}^{h}+K_{B}^{h}\right) \bar{A}_{2}^{h}-K_{2} \bar{A}_{2}=R_{2} .
\end{aligned}
$$

According to (15a) and (15b), $\bar{A}_{1}, \bar{A}_{2}, \kappa_{1}$, and $\kappa_{2}$ depend on the unknowns, $K_{1}$ and $K_{2}$, respectively. Hence, (18a) and (18b) can be rewritten as

$$
\begin{aligned}
& \sum_{h=1,3,5, \ldots}^{H}\left(K_{A}^{h}-K_{B}^{h}\right) \bar{A}_{1}^{h}\left(K_{1}\right)-K_{1} \bar{A}_{1}\left(K_{1}\right)=R_{1}\left(K_{1}\right), \\
& \sum_{h=1,3,5, \ldots}^{H}\left(K_{A}^{h}+K_{B}^{h}\right) \bar{A}_{2}^{h}\left(K_{2}\right)-K_{2} \bar{A}_{2}\left(K_{2}\right)=R_{2}\left(K_{2}\right) .
\end{aligned}
$$

Let $\bar{K}_{1}$ and $\bar{K}_{2}$ be the optimum values for the minimization of the overall harmonic residual squares in (19a) and (19b). Hence, the periods of the large amplitude modal vibrations are given by

$$
\begin{aligned}
& T_{1}\left(\bar{K}_{1}\right) \\
& =\frac{2 \pi}{\omega_{1}} \\
& =\frac{4}{\sqrt{\omega_{0}^{2}+\left(\left(\bar{K}_{1}+\beta A_{1}^{2}\right) / \rho\right)}} \int_{0}^{\pi / 2} \frac{1}{\sqrt{1-\kappa_{1}\left(\bar{K}_{1}\right)^{2} \sin (\psi)}} d \psi,
\end{aligned}
$$

$$
T_{2}\left(\bar{K}_{2}\right)
$$

$$
\begin{aligned}
& =\frac{2 \pi}{\omega_{2}} \\
& =\frac{4}{\sqrt{\omega_{0}^{2}+\left(\left(\bar{K}_{2}+\beta A_{2}^{2}\right) / \rho\right)}} \int_{0}^{\pi / 2} \frac{1}{\sqrt{1-\kappa_{1}\left(\bar{K}_{2}\right)^{2} \sin (\psi)}} d \psi,
\end{aligned}
$$

where $\omega_{1}$ and $\omega_{2}$ are the natural frequencies of the two nonlinear modes.

\section{Results and Discussions}

Using (20a) and (20b), the resonant frequencies of the inphase and out of phase modes of simply supported double panels can be obtained for various vibration amplitude ratios. Each double panel is made of two aluminum panels measuring $0.3048 \mathrm{~m} \times 0.3048 \mathrm{~m} \times 1.2192 \mathrm{~mm}$. The material properties are Young's modulus $E=7 \times 10^{10} \mathrm{~N} / \mathrm{m}^{2}$, Poisson's ratio $\nu=0.3$, and mass density $\rho=2700 \mathrm{~kg} / \mathrm{m}^{3}$. In Tables 1 (a) and 1(b), the first 25 symmetrical acoustic modes and the first four harmonic terms are used for the convergence checks of the resonant frequencies of the in-phase and out of phase modes. The frequency ratio is defined as $\omega_{n} / \omega_{o}$. It is shown that the nine acoustic modes and two harmonic terms approach is good enough for a converged and accurate frequency solution. Figures 2(a) and 2(b) show the comparisons between the free vibration time histories of the in-phase and out of phase mode vibrations obtained from the present 


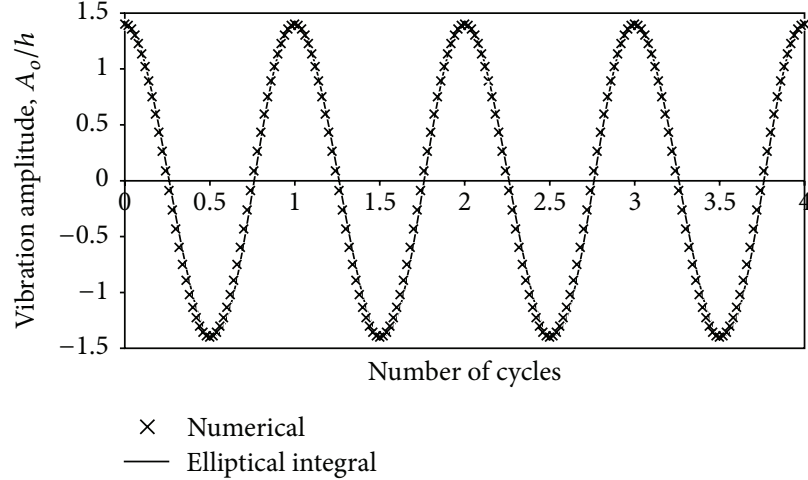

(a)

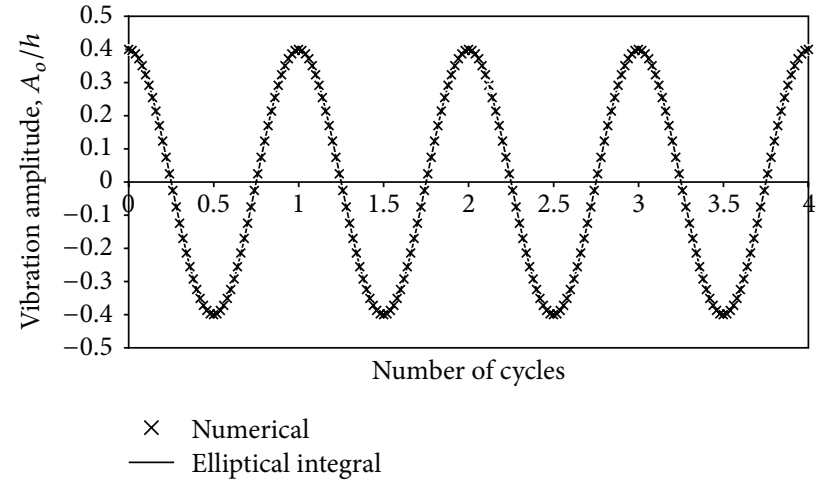

(b)

FIGURE 2: (a) Free vibration time histories obtained from the two solution methods (in-phase mode, initial amplitude ratio = 1.4, gap width/panel width $=0.5$ ). (b) Free vibration time histories obtained from the two solution methods (out of phase mode, initial amplitude ratio $=0.4$, gap width/panel width $=0.5$ ).

TABle 1: (a) Acoustic mode and harmonic convergences of the frequency ratio of an aluminum plate (in-phase mode, vibration amplitude/thickness =1). (b) Acoustic mode and harmonic convergences of the frequency ratio of an aluminum plate (out of phase mode, vibration amplitude/thickness $=1$ ).

(a)

\begin{tabular}{lcccc}
\hline$\omega_{n} / \omega_{o}$ & $\begin{array}{c}\text { Number of } \\
\text { harmonic } \\
\text { terms }=1\end{array}$ & 2 & 3 & 4 \\
\hline $\begin{array}{l}\text { Number of } \\
\text { acoustic modes }\end{array}$ & 1.3981 & 1.3981 & 1.3981 & 1.3981 \\
$=1$ & 1.3961 & 1.3962 & 1.3962 & 1.3962 \\
4 & 1.3961 & 1.3961 & 1.3961 & 1.3961 \\
9 & 1.3961 & 1.3961 & 1.3961 & 1.3961 \\
16 & 1.3961 & 1.3961 & 1.3961 & 1.3961 \\
25 & & & & \\
\hline
\end{tabular}

(b)

\begin{tabular}{lcccc}
\hline$\omega_{n} / \omega_{o}$ & $\begin{array}{c}\text { Number of } \\
\text { harmonic } \\
\text { terms }=1\end{array}$ & 2 & 3 & 4 \\
\hline $\begin{array}{l}\text { Number of } \\
\text { acoustic modes }\end{array}$ & 2.9676 & 2.9676 & 2.9676 & 2.9676 \\
$=1$ & 2.9505 & 2.9505 & 2.9505 & 2.9505 \\
4 & 2.9502 & 2.9502 & 2.9502 & 2.9502 \\
9 & 2.9502 & 2.9502 & 2.9502 & 2.9502 \\
16 & 2.9502 & 2.9502 & 2.9502 & 2.9502 \\
25 & & & & \\
\hline
\end{tabular}

elliptical integral method and numerical integration method [36]. It can be seen that the two sets of time histories are well agreed with each other.

In Figures 3-6, the cases of vibration amplitude $=0$ represent the results of linear vibration. It is because the nonlinear stiffness term is zero (in the linear vibration theory, there is no nonlinear term in the governing equation).
In Figures 3(a) and 3(b), the vibration amplitude ratios are plotted against the frequency ratios of the in-phase and out of phase mode vibrations for various gap widths. The aspect ratio is 1 . In Figure 3(a), when the vibration amplitude $=0$, the frequency ratio $=1$ (it is purely linear vibration. In the linear vibration, it is assumed that the vibration amplitude is very small); when the vibration amplitude $=1.4$, the frequency ratio is about 1.7 (it is highly nonlinear vibration). The natural frequency increases by $70 \%$ due to the nonlinear stiffness caused by the axial deformation along the panel (see Figure 3(c) for further explanation). The frequency ratios of the in-phase mode vibration are not very sensitive to the gap width (the three curves almost exactly coincide) while they are quite sensitive to and monotonically increasing with the vibration amplitude. When the amplitude is small, the frequency ratios increase very slowly. When the amplitude is large, the increase rates of the frequency ratios are almost constant. Note that the cases of infinite gap width in Figures 3(a) and 3(b) can be considered as a single panel without any gap effect. When compared with those in Figure 3(a), the frequency ratios of the out of phase mode vibration show higher degree of sensitivity to the gap width and lower degree of sensitivity to the vibration amplitude, respectively.

In Figures 4(a) and 4(b), the inverses of the gap width/ panel width, $a / c$, are plotted against the frequency ratios of the in-phase and out of phase mode vibrations for various vibration amplitude ratios. The aspect ratio is 1. In Figure 4(a), the frequency ratios of the in-phase mode vibration is almost unchanged for $a / c>0.3$. It is implied that the effect of $a / c$ is not significant on the frequency ratios for $a / c>0.3$. In the case of amplitude ratio $=1.4$, the frequency ratio significantly increases when $a / c<0.3$. Relatively, the frequency ratio of amplitude ratio $=1.4$ is slightly more sensitive than that of amplitude ratio $=0$. In Figure 4(b), the frequency ratios of the out of phase mode vibration are sensitive to $a / c$ when compared with those in Figure 4(a). Again, it can be seen that the higher the vibration amplitude ratio is, the higher the frequency ratio is. Besides, the slopes of the three curves, which represent the cases of amplitude ratio $=0,1,1.4$, are 


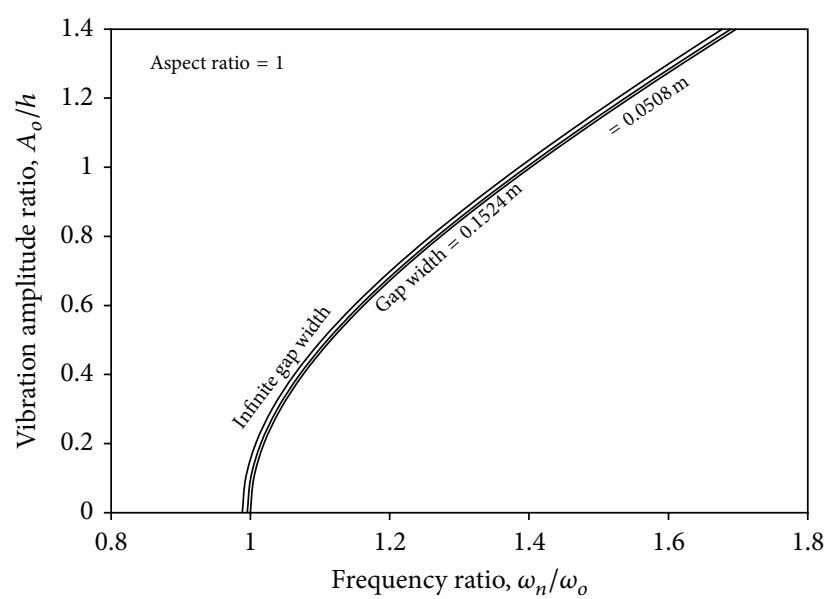

(a)

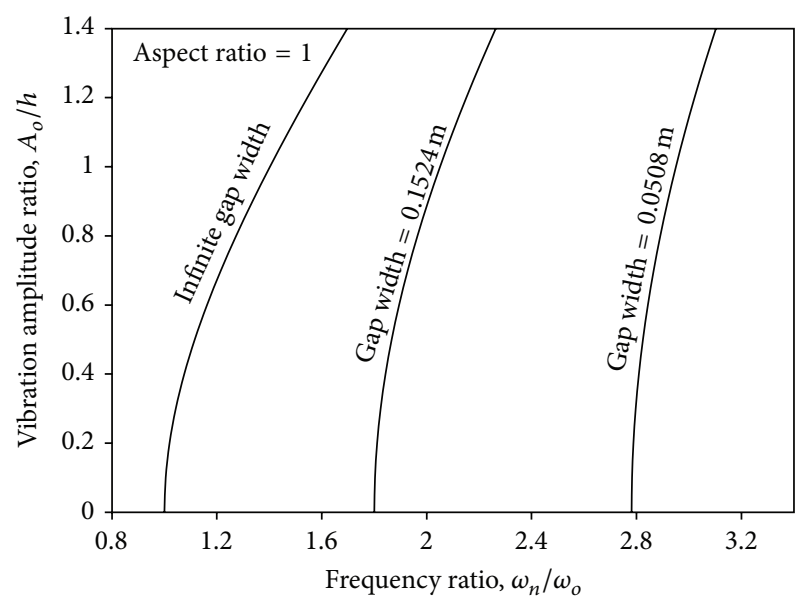

(b)

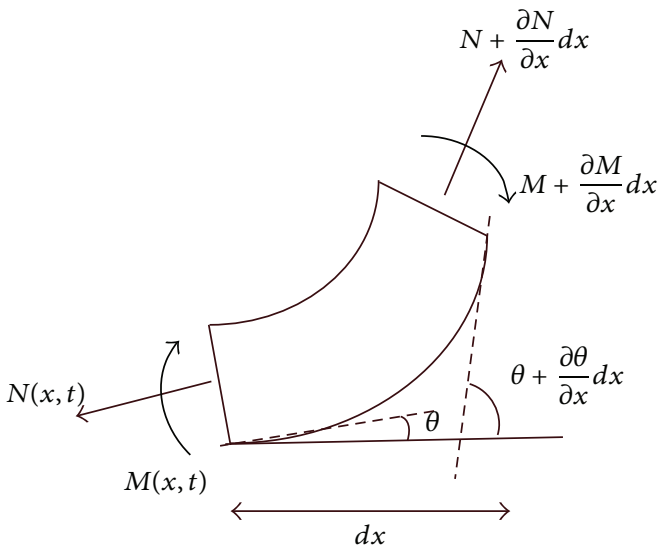

(c)

Figure 3: (a) Vibration amplitude ratio versus frequency ratio (in-phase mode, nine acoustic modes, two harmonic terms). (b) Vibration amplitude ratio versus frequency ratio (out of phase mode, nine acoustic modes, two harmonic terms). (c) Axial forces and moments acting on a plate element (side view). When the vibration amplitude is large, $\theta$ is large, the axial force acting on the panel is significant, and the nonlinear stiffness due to the axial deformation must be considered. When the vibration amplitude is small, $\sin \theta \approx 0$ and the axial force is neglectable (the linear vibration theory is valid for small deflection), where $N$ is the axial force; $M$ is the bending moment; $\theta$ is the slope.

almost the same. In Figure 4(c), it can be seen that the "net cavity volume change" of the in-phase mode vibration is smaller than that of the out of phase mode vibration. The amount of the volume change is larger and the acoustic stiffness is higher when the vibration amplitude is larger. Relatively, the effect of vibration amplitude on the natural frequency is relatively smaller when the acoustic stiffness is higher. That is why the frequency ratio of the in-phase mode vibration is less sensitive.

In Figures 5(a) and 5(b), the aspect ratios, $a / b$, are plotted against the frequency ratios of the in-phase and out of phase mode vibrations for various vibration amplitude ratios. The gap width is $0.0508 \mathrm{~m}$. In Figure 5(a), the frequency ratios of the in-phase mode vibration are almost constant for the zero vibration amplitude or $a / b>0.8$ in the cases of amplitude ratio $=1$ and 1.4. They are almost linearly decreasing with the aspect ratio for $a / b<0.8$. The effect of vibration amplitude is larger when the aspect ratio is smaller. In Figure 5(b), the frequency ratio of the out of phase mode is almost constant for $a / b<0.25$. They are increasing with the aspect ratio for the cases of $a / b>0.25$. Relatively, they are more sensitive to the aspect ratio than those of the in-phase mode vibration. When the aspect ratio is larger, the differences between the three frequency ratio curves are smaller (i.e., the effect of vibration amplitude is smaller).

In Figures 6(a)-6(d), the vibration amplitudes are plotted against the acoustic stiffness ratios of the in-phase and out of phase modes for various gap widths. The aspect ratio is 1 . Note that $\left(K_{A}^{1}-K_{B}^{1}\right)$ and $\left(K_{A}^{1}+K_{B}^{1}\right)$ are defined as $K_{\text {air }}$ in Figures 6(a) and 6(b). In Figure 6(a), the acoustic stiffness ratio of the in-phase mode is almost constant for $A_{o} / h<0.25$, while it is nonlinearly decreasing with the vibration amplitude for $A_{o} / h>0.25$. The physical meaning of negative acoustic stiffness is that the acoustic force direction is the same as the panel vibration direction. Besides, when the gap is small, the acoustic stiffness is strong (it can be imagined that a larger force is required to compress a smaller cavity). Thus, the effect of vibration amplitude on the natural frequency 


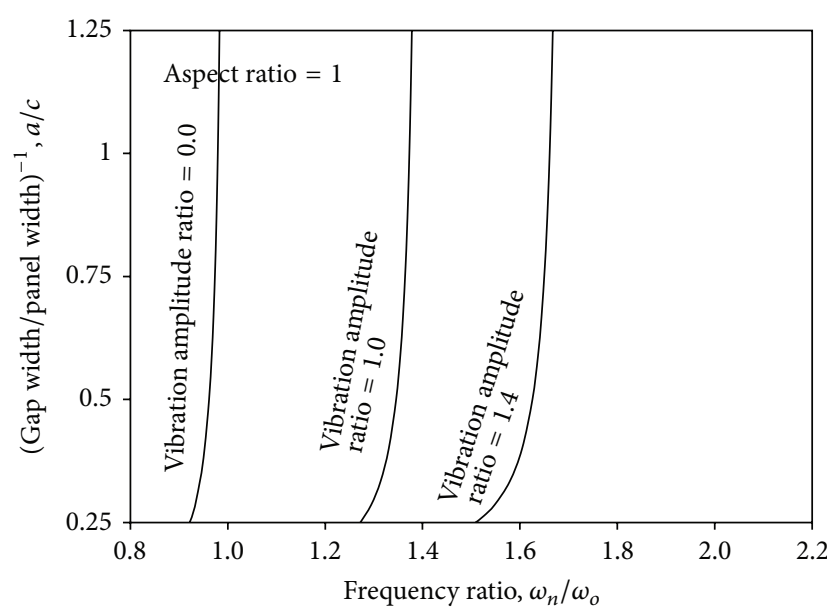

(a)

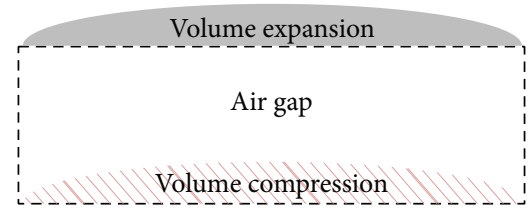

In-phase mode

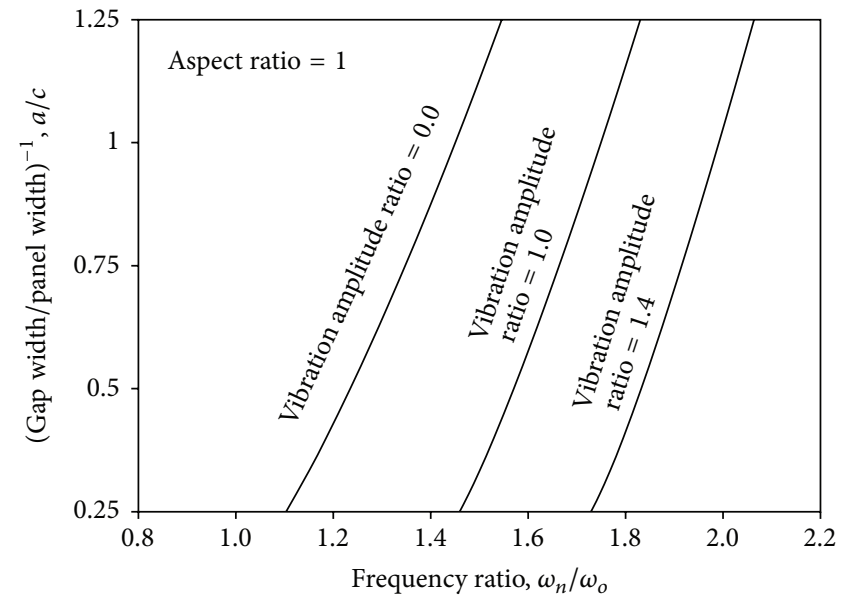

(b)

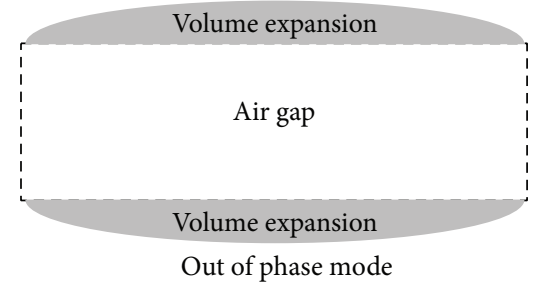

Figure 4: (a) (Gap width/panel width) ${ }^{-1}$ versus frequency ratio (in-phase mode, nine acoustic modes, two harmonic terms). (b) (Cavity depth/width) $)^{-1}$ versus frequency ratio (out of phase mode, nine acoustic modes, two harmonic terms). (c) Volume changes of the in-phase and out of phase modes.

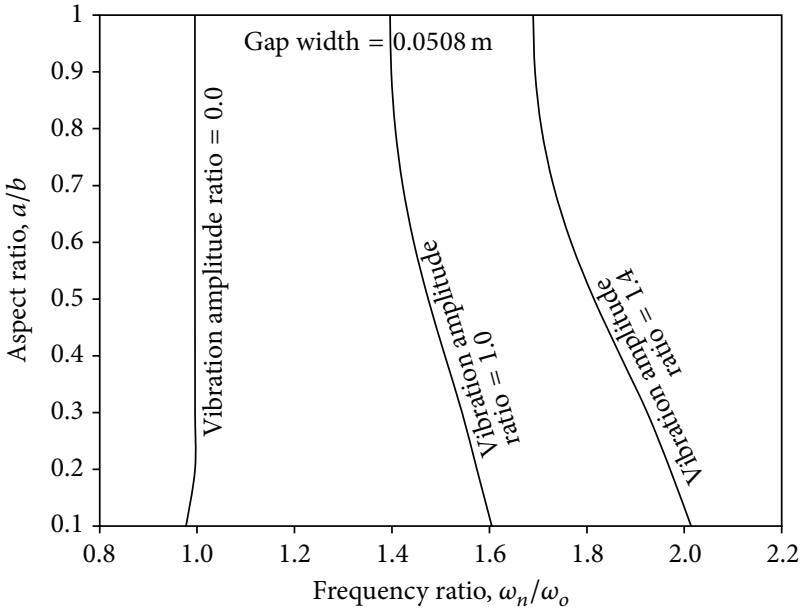

(a)

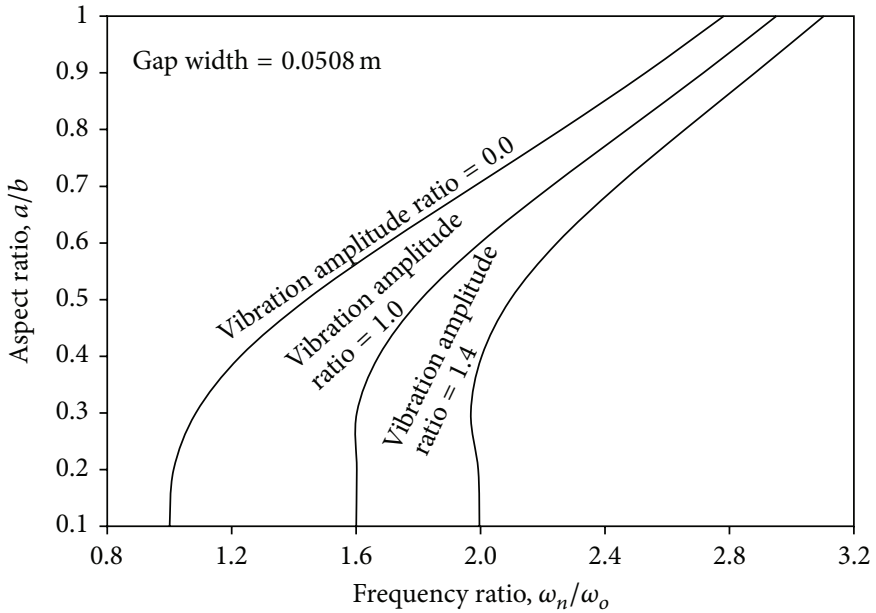

(b)

FIGURE 5: (a) Aspect ratio versus frequency ratio (in-phase mode, nine acoustic modes, two harmonic terms). (b) Aspect ratio versus frequency ratio (out of phase mode, nine acoustic modes, two harmonic terms).

is relatively smaller when the gap width is smaller (or the acoustic stiffness is higher). That is why the acoustic stiffness of large gap width in Figure 6(a) is more sensitive than that of small gap width. Unlike those in Figure 6(a), the three curves in Figures 6(b)-6(d) are far from each other (that is why they are plotted in three graphs). The slopes of the three curves are similar to each other, and the acoustic stiffness vales are nonlinearly decreasing with the vibration amplitude. Generally, the acoustic stiffness of the out of phase mode vibration is less sensitive to the vibration amplitude than that of the in-phase mode vibration. It is because the acoustic stiffness value of the out of phase mode vibration, $\left(K_{A}^{1}+K_{B}^{1}\right)$, 


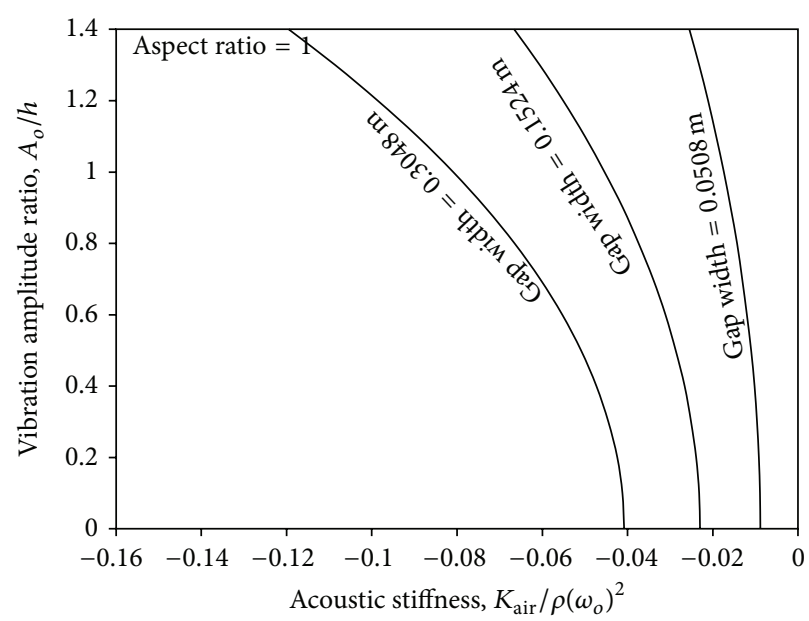

(a)

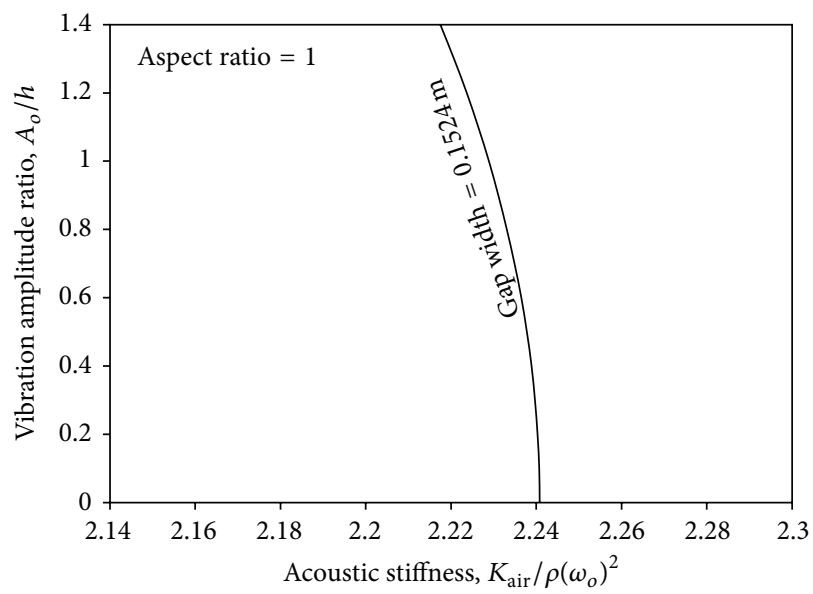

(c)

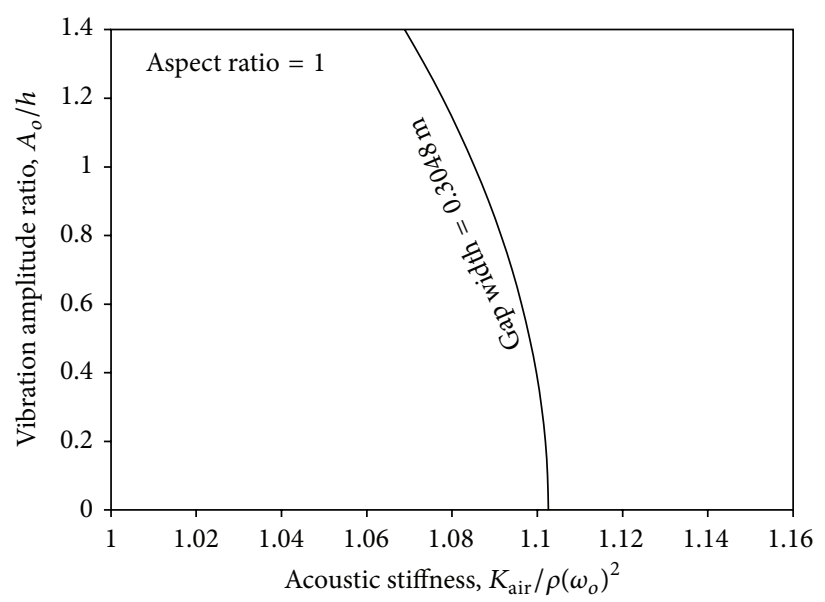

(b)

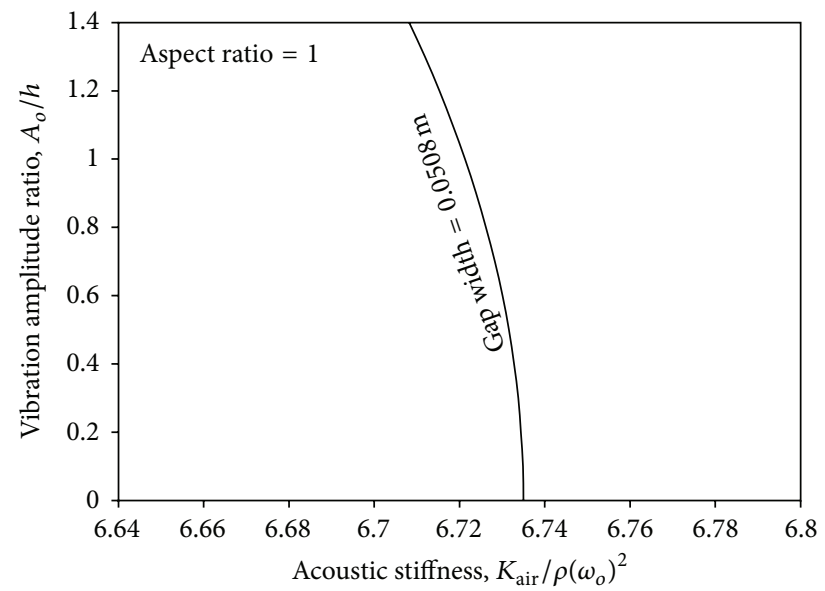

(d)

FIGURE 6: (a) Vibration amplitude ratio versus acoustic stiffness (in-phase mode, nine acoustic modes, two harmonic terms). (b) Vibration amplitude ratio versus acoustic stiffness (gap width $=0.3048 \mathrm{~m}$, out of phase mode, nine acoustic modes, two harmonic terms). (c) Vibration amplitude ratio versus acoustic stiffness (gap width $=0.1524 \mathrm{~m}$, out of phase mode, nine acoustic modes, two harmonic terms). (d) Vibration amplitude ratio versus acoustic stiffness (gap width $=0.0508 \mathrm{~m}$, out of phase mode, nine acoustic modes, two harmonic terms).

is higher than that of in-phase mode vibration, $\left(K_{A}^{1}-K_{B}^{1}\right)$. As aforementioned, the effect of vibration amplitude on the natural frequency is relatively smaller when the acoustic stiffness is higher.

From Figure 6(a), it is known that the acoustic stiffness values would be negative and the frequency ratios in some cases could be less than 1 (see Figures 5(a) and 5(b)). Unlike the acoustic stiffness, the nonlinear structural stiffness is always positive and nonlinearly increasing with the vibration amplitude (see Figure 7). It is noted that the nonlinear stiffness is zero when the vibration amplitude is zero (in the linear vibration, it is assumed that the vibration amplitude is very small). When the vibration amplitude is small, the slope of the curve representing the nonlinear stiffness is deep (it means that the nonlinear stiffness increases quickly). When the vibration amplitude is large, the slope is almost constant. The nonlinear stiffness constantly increases with the vibration amplitude.

\section{Conclusions}

A multistructural/acoustic mode formulation, which is based on the classical nonlinear plate equation and homogeneous wave equation, has been presented for the large amplitude free vibrations of a double panel. The present study proposes the elliptical integral method to obtain the natural frequencies of various nonlinear double panels. The effects of the gap width, aspect ratio, and vibration amplitude ratio on the resonant frequencies of the in-phase and out of phase modes have also been investigated. It has been found that (1) the present elliptic integral solutions agree reasonably well with those obtained from the numerical integration method, and they are convergent with increasing the numbers of acoustic modes and harmonic terms; (2) the frequency ratio of the inphase mode of a double panel is more like that of a single panel, and the frequency ratio of the out of phase mode is more sensitive to the gap width and aspect ratio; (3) the acoustic stiffness of the in-phase mode is more sensitive to 


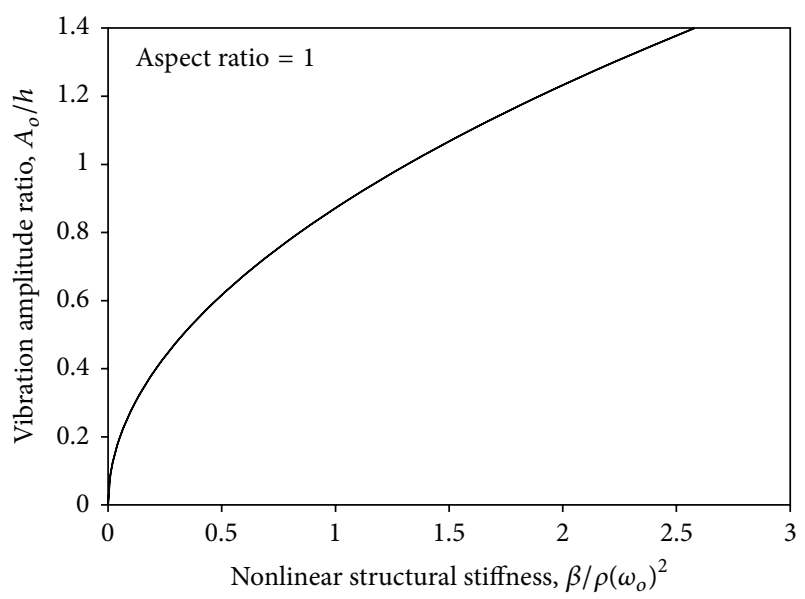

FIGURE 7: Vibration amplitude ratio versus structural nonlinear stiffness.

the vibration amplitude than that of the out of phase mode; and (4) unlike the structural stiffness, the acoustic stiffness of a double panel may be negative and depends on the vibration amplitude.

In practice, when acoustic engineers design a double panel used for noise reduction, they would set the natural frequency not overlapping with the dominant noise frequency bandwidth. Thus, the natural frequency results in this study can be used as reference in noise reduction panel design.

\section{Conflict of Interests}

The author declares that there is no conflict of interests regarding the publication of this paper.

\section{Acknowledgments}

The work described in this paper was fully supported by a grant from the Research Grants Council of Hong Kong (project title: A New Solution Method to Nonlinear PanelCavity Coupling Problems: Multilevel Residue Harmonic Balance Method). The author would like to express his gratitude and appreciation to Dr. W. Y Poon's advices and contributions.

\section{References}

[1] A. London, "Transmission of reverberant sound through double walls," The Journal of the Acoustical Society of America, vol. 22, no. 2, pp. 270-279, 1950.

[2] A. Cummings and K. A. Mulholland, "The transmission loss of finite sized double panels in a random incidence sound field," Journal of Sound and Vibration, vol. 8, no. 1, pp. 126-133, 1968.

[3] A. J. Price and M. J. Crocker, "Sound transmission through double panels using statistical energy analysis," The Journal of the Acoustical Society of America, vol. 47, no. 3, pp. 683-693, 1970.

[4] F. X. Xin and T. J. Lu, "Analytical and experimental investigation on transmission loss of clamped double panels: implication of boundary effects," Journal of the Acoustical Society of America, vol. 125, no. 3, pp. 1506-1517, 2009.

[5] F. X. Xin and T. J. Lu, "Sound radiation of orthogonally ribstiffened sandwich structures with cavity absorption," Composites Science and Technology, vol. 70, no. 15, pp. 2198-2206, 2010.

[6] F. X. Xin and T. J. Lu, "Transmission loss of orthogonally rib-stiffened double-panel structures with cavity absorption," Journal of the Acoustical Society of America, vol. 129, no. 4, pp. 1919-1934, 2011.

[7] F. X. Xin and T. J. Lu, "Analytical modeling of sound transmission through clamped triple-panel partition separated by enclosed air cavities," European Journal of Mechanics, A/Solids, vol. 30, no. 6, pp. 770-782, 2011.

[8] F. X. Xin, T. J. Lu, and C. Q. Chen, "Vibroacoustic behavior of clamp mounted double-panel partition with enclosure air cavity," Journal of the Acoustical Society of America, vol. 124, no. 6, pp. 3604-3612, 2009.

[9] F. X. Xin, T. J. Lu, and C. Q. Chen, "Sound transmission through simply supported finite double-panel partitions with enclosed air cavity," Journal of Vibration and Acoustics, Transactions of the ASME, vol. 132, no. 1, Article ID 011008, 2010.

[10] F. X. Xin, T. J. Lu, and C. Q. Chen, "External mean flow influence on noise transmission through double-leaf aeroelastic plates," AIAA Journal, vol. 47, no. 8, pp. 1939-1951, 2009.

[11] J. Pan and C. Bao, "Analytical study of different approaches for active control of sound transmission through double walls," The Journal of the Acoustical Society of America, vol. 103, no. 4, pp. 1916-1922, 1998.

[12] O. E. Kaiser, S. J. Pietrzko, and M. Morari, "Feedback control of sound transmission through a double glazed window," Journal of Sound and Vibration, vol. 263, no. 4, pp. 775-795, 2003.

[13] S. Langer and H. Antes, "Analyses of sound transmission through windows by coupled finite and boundary element methods," Acta Acustica, vol. 89, no. 1, pp. 78-85, 2003.

[14] Y. Y. Lee and C. F. Ng, "Sound insertion loss of stiffened enclosure plates using the finite element method and the classical approach," Journal of Sound and Vibration, vol. 217, no. 2, pp. 239-260, 1998.

[15] Y. Y. Lee and E. W. M. Lee, "Widening the sound absorption bandwidths of flexible micro-perforated curved absorbers using structural and acoustic resonances," International Journal of Mechanical Sciences, vol. 49, no. 8, pp. 925-934, 2007.

[16] F. Alijani and M. Amabili, "Chaotic vibrations in functionally graded doubly curved shells with internal resonance," International Journal of Structural Stability and Dynamics, vol. 12, no. 6, Article ID 1250047, 23 pages, 2012.

[17] C. S. Chen, C. W. Chen, and W. R. Chen, "Dynamic stability characteristics of functionally graded plates under arbitrary periodic loads," International Journal of Structural Stability and Dynamics, vol. 13, no. 6, Article ID 1350026, 2013.

[18] D. K. Nguyen, "A Timoshenko beam element for large displacement analysis of planar beams and frames," International Journal of Structural Stability and Dynamics, vol. 12, no. 6, Article ID 1250048, 2012.

[19] V. Dogan and R. Vaicaitis, "Nonlinear response of double-wall cylindrical shell vibrations under random excitation," Journal of Aerospace Engineering, vol. 19, no. 1, pp. 46-54, 2006.

[20] Y. Wei and R. Vaicaitis, "Nonlinear models for double-wall systems for vibrations and noise control," Journal of Aircraft, vol. 34, no. 6, pp. 802-810, 1997. 
[21] Y. Y. Lee, "Structural-acoustic coupling effect on the nonlinear natural frequency of a rectangular box with one flexible plate," Applied Acoustics, vol. 63, no. 11, pp. 1157-1175, 2002.

[22] Y. Y. Lee, X. Guo, and E. W. M. Lee, "Effect of the large amplitude vibration of a finite flexible micro-perforated panel absorber on sound absorption," International Journal of Nonlinear Sciences and Numerical Simulation, vol. 8, no. 1, pp. 41-44, 2007.

[23] C. K. Hui, Y. Y. Lee, and J. N. Reddy, "Approximate elliptical integral solution for the large amplitude free vibration of a rectangular single mode plate backed by a multi-acoustic mode cavity," Thin-Walled Structures, vol. 49, no. 9, pp. 1191-1194, 2011.

[24] Y. Y. Lee, Q. S. Li, A. Y. T. Leung, and R. K. L. Su, “The jump phenomenon effect on the sound absorption of a nonlinear panel absorber and sound transmission loss of a nonlinear panel backed by a cavity," Nonlinear Dynamics, vol. 69, no. 1-2, pp. 99116, 2012.

[25] H. N. Chu and G. Herrmann, "Influence of large amplitudes on free flexural vibrations of rectangular elastic plates," Journal of Applied Mechanics, vol. 23, pp. 532-540, 1956.

[26] C. Yang, "Approximate super- and sub-harmonic response of a multi-DOFs system with local cubic nonlinearities under resonance," Journal of Applied Mathematics, vol. 2012, Article ID 531480, 22 pages, 2012.

[27] Z. Zhou-Lian, L. Chang-Jiang, H. Xiao-Ting, and C. Shan-Lin, "Free vibration analysis of rectangular orthotropic membranes in large deflection," Mathematical Problems in Engineering, vol. 2009, Article ID 634362, 9 pages, 2009.

[28] R. A. Jafari-Talookolaei, M. H. Kargarnovin, M. T. Ahmadian, and M. Abedi, "An investigation on the nonlinear free vibration analysis of beams with simply supported boundary conditions using four engineering theories," Journal of Applied Mathematics, vol. 2011, Article ID 842805, 17 pages, 2011.

[29] Y. Y. Lee, W. Y. Poon, and C. F. Ng, "Anti-symmetric mode vibration of a curved beam subject to autoparametric excitation," Journal of Sound and Vibration, vol. 290, no. 1-2, pp. 48-64, 2006.

[30] Y. Y. Lee, J. L. Huang, C. K. Hui, and C. F. Ng, “Sound absorption of a quadratic and cubic nonlinearly vibrating curved panel absorber," Applied Mathematical Modelling, vol. 36, no. 11, pp. 5574-5588, 2012.

[31] W. Y. Poon, C. F. Ng, and Y. Y. Lee, "Dynamic stability of a curved beam under sinusoidal loading," Proceedings of the Institution of Mechanical Engineers G: Journal of Aerospace Engineering, vol. 216, no. 4, pp. 209-217, 2002.

[32] R. Soroush, A. Koochi, A. S. Kazemi, and M. Abadyan, "Modeling the effect of van der waals attraction on the instability of electrostatic cantilever and doubly-supported nano -beams using modified a domian method," International Journal of Structural Stability and Dynamics, vol. 12, no. 5, Article ID 1250036, 2012.

[33] I. V. Andrianov, V. I. Olevs'kyy, and J. Awrejcewicz, "Analytical perturbation method for calculation of shells based on 2D Pade approximants," International Journal of Structural Stability and Dynamics, vol. 13, no. 7, Article ID 1340003, 2013.

[34] M. Gu and S.-Y. Ren, "Parametric vibration of stay cables under axial narrow-band stochastic excitation," International Journal of Structural Stability and Dynamics, vol. 13, no. 8, Article ID 1350035, 2013.

[35] S.-H. Yin, "A new explicit time integration method for structural dynamics," International Journal of Structural Stability and Dynamics, vol. 13, no. 3, Article ID 1250068, 23 pages, 2013.
[36] Y. Y. Lee, H. Y. Sun, and J. N. Reddy, "Non-linear finite element modal approach for the large amplitude free vibration of symmetric and unsymmetric composite plates," International Journal for Numerical Methods in Engineering, vol. 65, no. 1, pp. 45-61, 2006.

[37] R. Ma, J. Li, and C. Gao, "Existence of positive solutions of a discrete elastic beam equation," Discrete Dynamics in Nature and Society, vol. 2010, Article ID 582919, 15 pages, 2010.

[38] S. L. Zhu and C. Sheng, "Oscillation and nonoscillation criteria for nonlinear dynamic systems on time scales," Discrete Dynamics in Nature and Society, vol. 2012, Article ID 137471, 14 pages, 2012.

[39] W. Chen, Z. Han, S. Sun, and T. Li, "Oscillation behavior of a class of second-order dynamic equations with damping on time scales," Discrete Dynamics in Nature and Society, vol. 2010, Article ID 907130, 15 pages, 2010. 


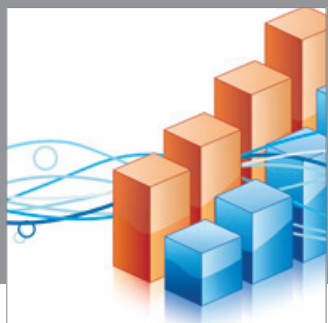

Advances in

Operations Research

mansans

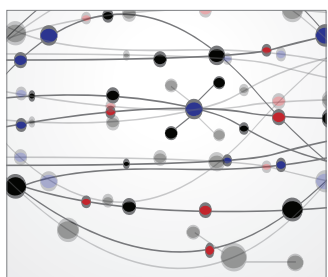

The Scientific World Journal
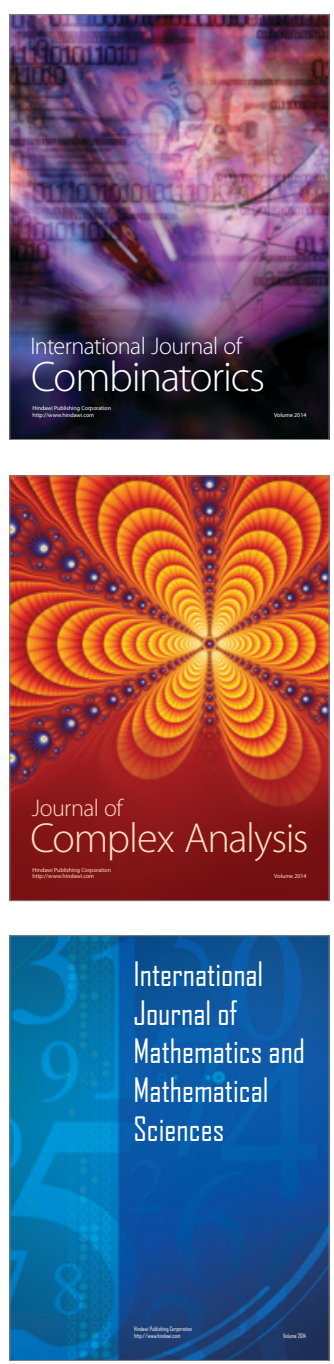
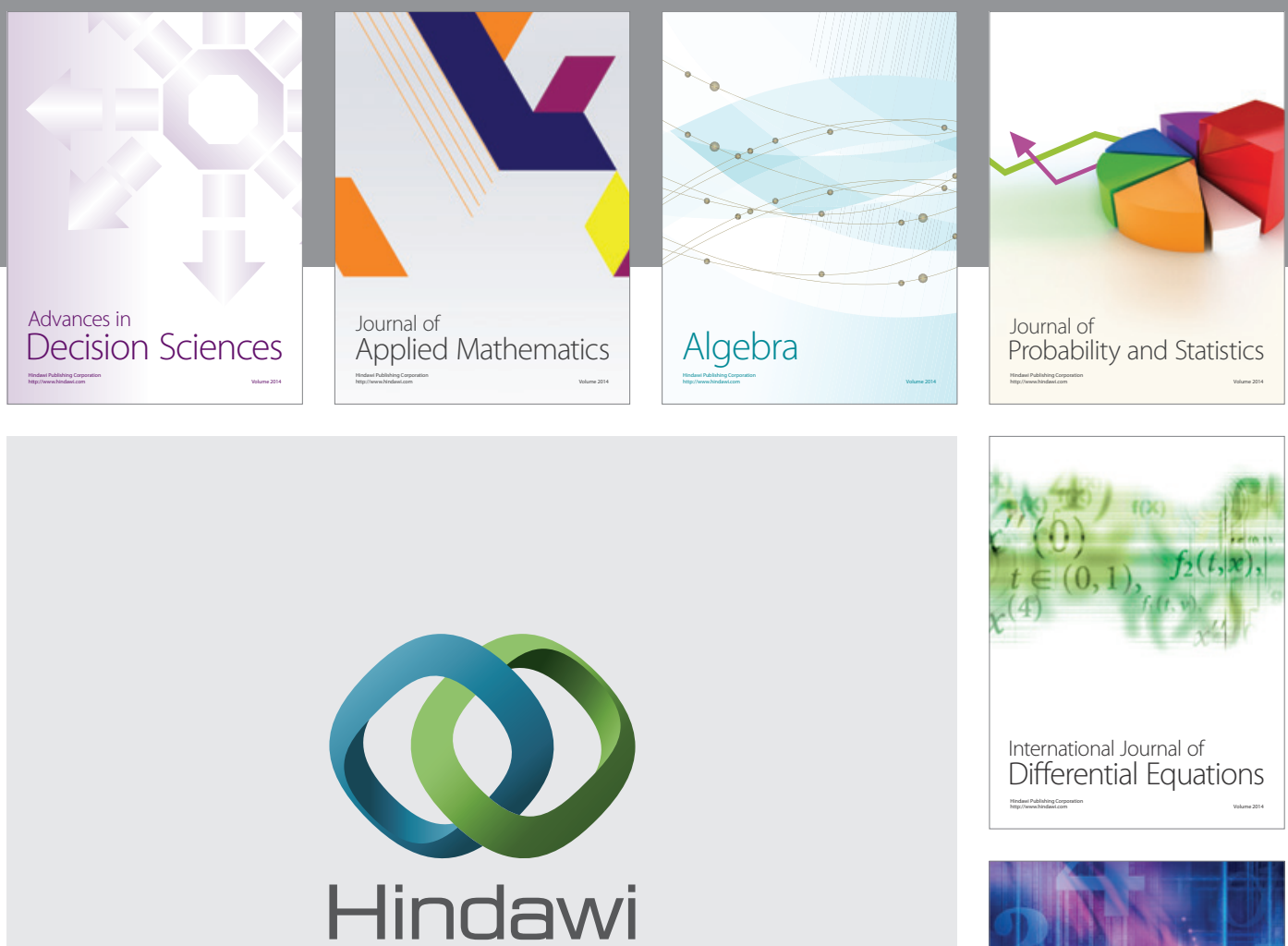

Submit your manuscripts at http://www.hindawi.com
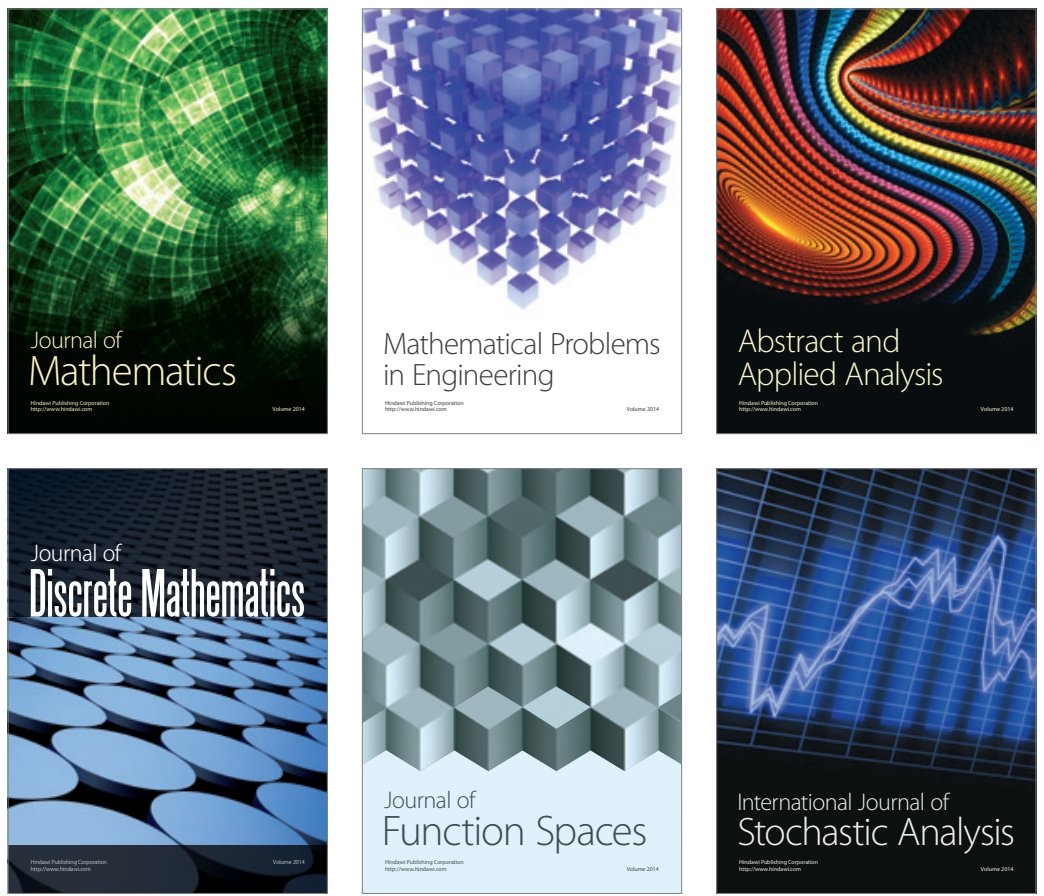

Journal of

Function Spaces

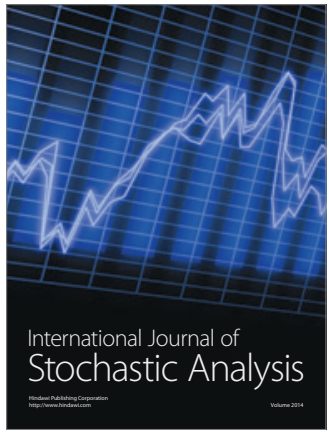

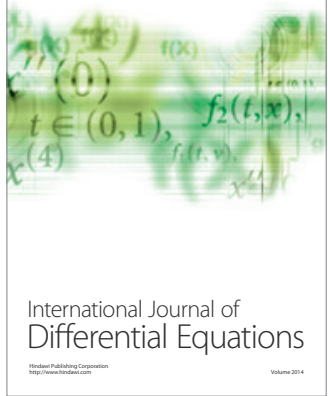
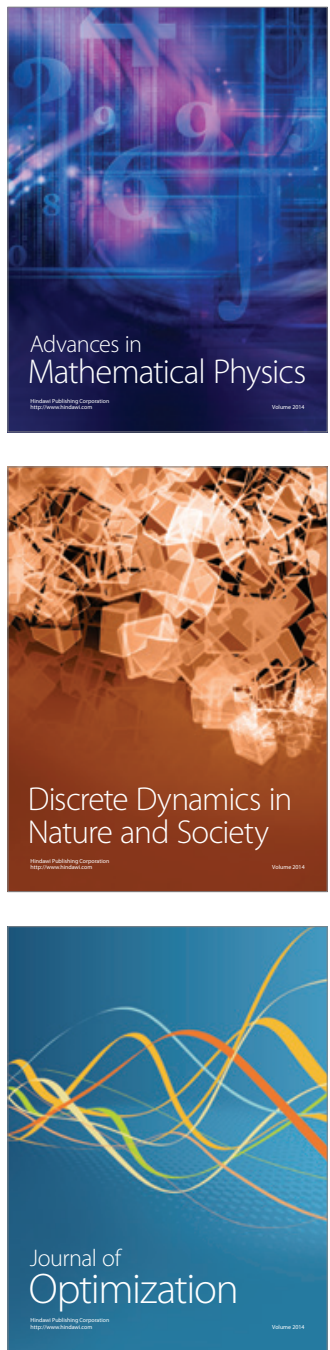